\title{
New Clustering Topology for Wireless Sensor Networks
}

\author{
Mei $\mathrm{Hu}^{1, \text { a }}$,Yongxi Wang ${ }^{1, \mathrm{~b}}$ \\ ${ }^{1}$ School of Electronic and Information Engineering, Lanzhou Institute of Technology, Lanzhou \\ 730050, China.
}

Keywords: farmland, Hexi corridor, Ad-hoc network, two-level, linear, clustering, energy-efficient.

\begin{abstract}
According to the characteristic of farmland topography in the Hexi corridor, the distributed two-level linear cluster topology is built. The protocol improves the way of the random method of the nodes distribution. The cluster nodes of the irrigation sub-network which have not been a cluster head can compete with the principle of equivalent possibility, and on the basis of the rest energy of nodes the cluster head is selected at last. Simulation experiment results show that the distributed two-level linear cluster protocol in the survival of nodes and the ratio of success are superior to the ad-hoc network.
\end{abstract}

\section{Introduction}

The Hexi corridor is located in the northwest of China, which belongs to the typical droughty desert environment and has the characteristics of can't grow without irrigation. Agriculture is the pillar industry of the area, also is the area of large water consumption. The area of farmland is divided by vast gobi and desert partition, which is discontinuous and strips. Information is accurately, all the day and continuously collected with Zigbee wireless sensor network under large area and complicated conditions. A sustainable development model is provided instead of the flood irrigation in vulgar irrigation method.

\section{Ad-hoc network topology of farmland}

Because of the farmland sheer size, diverse terrain and complicated surroundings in China, the sensor nodes in the farmland irrigation is large-scale randomly placed and no rule is follow. Terminal nodes in the topology are only responsible for their own coverage data, and then transmits the data to the routing node. Ad-hoc network topology of farmland is shown in fig.1.

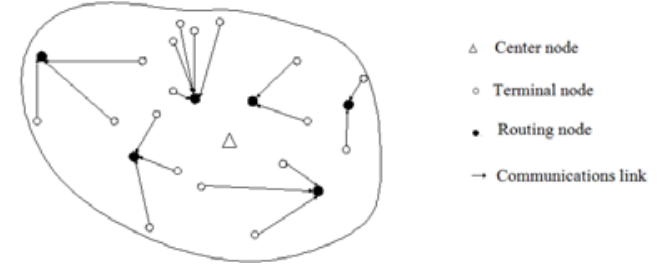

Fig.1 Ad-hoc network topology of farmland

The figure 1 shows that ad-hoc network topology of Zigbee nodes in farmland exists the following questions:

(1) The nodes are randomly placed in farmland. So it is probable to place node repeatedly or no one in a certain area, leading to the data redundancy rate high or miss information for some land.

(2) A large number of terminal nodes in network collect information from local, and then send to routing nodes. So at some time routing nodes receive a large amount of data, producing the strong data conflict and leading to decline of the whole network's throughput.

(3) As the protocol is static, it's routing nodes are fixed and immovable. Routing nodes send a mounts of data to the center node from start to finish. If the terminal nodes near routing nodes is randomly placed too much, routing nodes will die and lead to communication interruption of the region. 


\section{The Structure of system}

The farmland environment, terrain, and the actual irrigation infrastructure of the Hexi corridor are analyzed, while the research experience at home and abroad are learnt. In the end the whole irrigation area is divided into a two-level distributed topology. The whole system is consists of a number of irrigation subnets, remote data communication network (public mobile communication network or wired network) and the control center .

In our country, the drip irrigation in the Hexi corridor is vigorously supported by the government. Drip irrigation system is composed of main pump station, control valves, main pipe, branch pipe and dropper pipe.The triple pipes are generally laid in line. Combined with the strip topographic feature in Hexi corridor, the pipe laying in the farmland and the scope of terminal nodes gathering the data, the irrigation field is divided into a uniform plot (irrigation subnet) with $\mathrm{Z}$ width, $\mathrm{L}$ length. And then the $\mathrm{N}$ sensor nodes and miniature solenoid valves are linearly placed in each irrigation subnet. The solenoid valves can control the dropper on the branch, as shown in figure 2 . The $\mathrm{N}$ terminal nodes are linearly placed form a cluster in the irrigation subnet. There is a two-level cluster head, which is not fixed in each cluster. In the cluster the nodes compete with the principle of equivalent possibility and on the basis of the rest energy of nodes the cluster head is selected at last. According to the rule the topology with two-level clustering linear wireless sensor network is formed, as shown in figure 3. So energy load is evenly distributed to the terminal nodes of the cluster, balances energy consumption of the network and prolongs the average life of nodes. At last the success rate of data packet is improved.

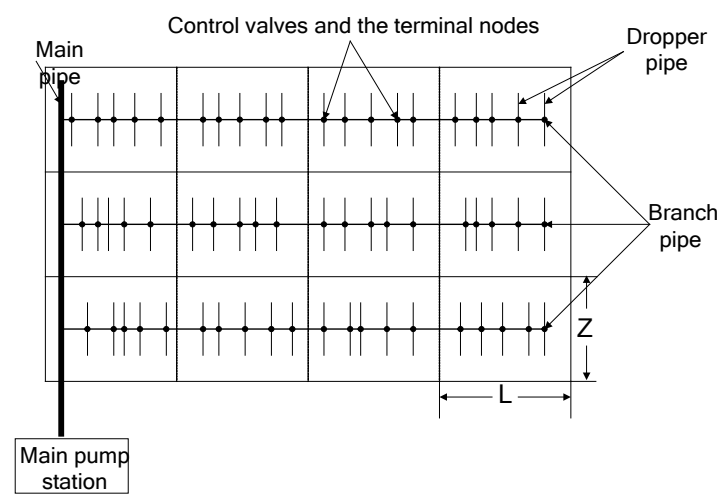

Fig.2 Dividing of irrigation field

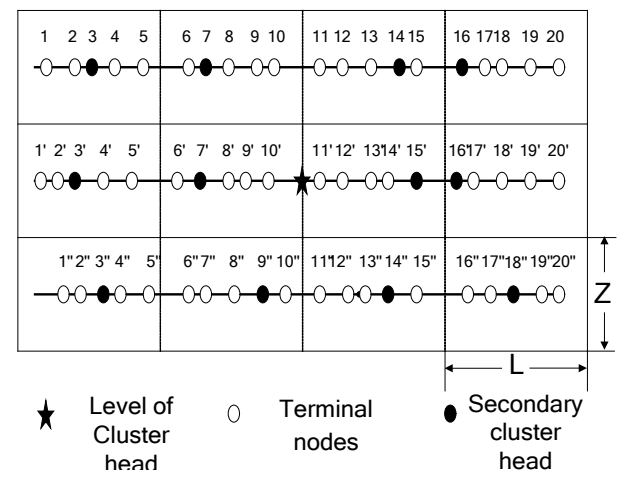

Fig.3 Topology of the irrigation subnet

\section{Algorithm of two-level linear cluster}

\subsection{Algorithm design in the cluster}

Ad-hoc network topology is essentially a simple LEACH (Low Energy Adaptive Clustering Hierarchy) algorithm, so it meets the characteristics of the LEACH algorithm. The LEACH algorithm generates a number between $0 \sim 1$ for each node randomly. If the random number by selected is less than a certain threshold T (n), the node becomes the cluster head. T (n) can be got by mathematical calculations, as shown in formula 1.

$$
T(\mathrm{n})= \begin{cases}\frac{1}{1-p^{*}\left[r^{*} \bmod (1 / p)\right]} & N \in G \quad(1) \\ 0 & \text { else }\end{cases}
$$

$\mathrm{P}$ is the percentage of cluster head in all network nodes in each round. $\mathrm{R}$ is the ongoing election round number. $\mathrm{G}$ is the set of which have not been a cluster head in the rest of 1 / p cycle.

The cluster head is elected randomly in the LEACH algorithm without considering the rest energy of nodes. Then it is possible to choose the node with the smaller remaining energy to be cluster head. But the cluster head likely dies leading to the communication interrupt of the cluster. The two-level linear clustering protocol is dynamic, as shown in figure 3. Terminal nodes in turn send real-time collected information to the two-level cluster head by TDMA mode within the cluster. A terminal node collects a frame of information in figure 3 , and then the information is transmitted to the 
two-level cluster head by single jump in line. Ultimately the information is sent to the level of cluster head by single jump or more than one hop. So the probability of the terminal nodes which become two-level cluster head is $\frac{1}{N}$ (assuming that all the nodes have the same initial energy). Similarly, when the other terminal nodes collects another frame of information, the remaining $\mathrm{N}-1$ terminal nodes which have not been the two-level cluster head compete with the equal probability of $\frac{1}{N-1}$.

Eventually on the basis of the current surplus energy the cluster head is selected. Thus the probability of which the terminal node with high energy becomes the two-level cluster head increases, and improves the robustness of the network. Therefore the probability that the terminal nodes become the two-level cluster head can be calculated according to the following formulas.

Introducing parameter $\varepsilon$, it is the current level of rest energy. $\varepsilon_{\mathrm{c}}$ is the rest energy in the current round. $\varepsilon_{\mathrm{o}}$ is the node initial energy, then:

$$
\varepsilon=\frac{\varepsilon_{\mathrm{c}}}{\varepsilon_{o}}
$$

Bringing the formula (2) into the formula (1),then the formula (3) can be get.

$$
T(\mathrm{n})= \begin{cases}\frac{1}{1-p^{*}\left[r^{*} \bmod (1 / p)\right]} * \varepsilon & N \in G \\ 0 & \text { else }\end{cases}
$$

So the energy load of cluster is evenly distributed to the terminal nodes, the death number of nodes is reduced, the average life of the cluster nodes is extended and the success rate of data packet is improved by rotating the two-level cluster head and considering the rest energy of the two-level cluster head.

\subsection{Algorithm design between cluster}

Routing between clusters refers to the two-level cluster head by single hop or multiple hops to reach the level of cluster head. The energy consumption of the level cluster head is very big, because the level cluster head receives data from the two-level cluster head and the control command from the level cluster head to the terminal nodes. Outside the cluster energy consumption is directly proportional to the square of the distance d. Combined with the strip topographic feature in Hexi corridor, the two-level cluster head communicates with the level cluster head through linear single hop or multiple hops. If the distance is short between the two-level cluster head and the level cluster head, one jump directly is adopted. But if the distance is far away, more than one jump can be used between the two-level cluster heads, and then the level of cluster head communication with one hop. Thus it ensures the phenomenon of network communication interruption don't take place because the energy of the level cluster head is too low. So the average life of the whole network is extended and the success rate of data packet is improved.

Between clusters data communication reduces the data redundancy for the level cluster head and improves the success rate of data packet by the method of data fusion. Data aggregation is carried out in accordance with the formula (4):

$$
N^{n} K \rightarrow K \quad(4)
$$

The $\mathrm{N}^{\mathrm{n}}$ is the number of all terminal nodes in irrigation field. The $\mathrm{K}$ is the packet length of the two-level cluster head transmitting to the level cluster head.

\section{Application model and the simulation results}

\subsection{Application model design}

The application model is a strip area with $1000 \mathrm{~m}$ length, $500 \mathrm{~m}$ width in NS2 simulation software. The level cluster head is located in the geometric center of the region, the coordinates of $(500,250)$, as shown in figure3.The area is divided into 12 equal irrigation subnet and the five terminal nodes is linearly placed in each subnet. The location information of all nodes is known. Initial energy of the network nodes is $2 \mathrm{~J}$, and packet size is 525 byte. 
If the terminal node 1 collects a frame of information in irrigation subnet 1 , terminal node 2 communicates with the two-level cluster head 3 by more than one jump and it completes communication with base station in one hop. After a while terminal node 4 collects a new frame of information in the irrigation subnet, and then the terminal nodes 1, 2 and 5 which have not been the two-level cluster head become the new cluster head with a third of the probability. Finally they complete to be the cluster head according to their remaining energy.

\subsection{Simulation results}

Ad-hoc network topology and the two-level linear cluster protocol are respectively simulated. Compared with the ad-hoc network topology, the death nodes of the two-level cluster linear protocol decrease significantly in figure 5.The protocol uses competition with equal probability for the terminal nodes and the two-level cluster heads are the one which the rest energy is larger. Therefore it ensures that the entire network energy is in balance, the death nodes reduced and the life of the network prolongs.

The success rate of the data packets in the two-level linear cluster protocol is higher than the ad-hoc network topology in figure 6 . After the 2500s the success rate of the data packets goes down sharply in the ad-hoc network topology, but the two-level linear cluster protocol is not obvious. Data aggregation and the generation of two-level cluster head gradually reflect on the success rate of data packets with the network working time growing.

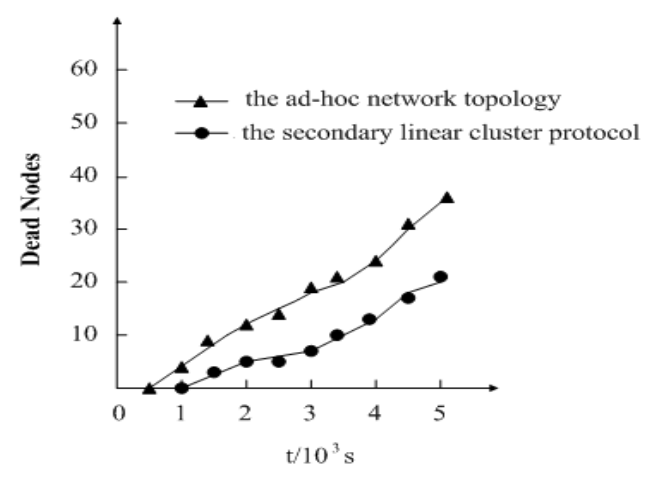

Fig.5 Dead nodes vs. network working time

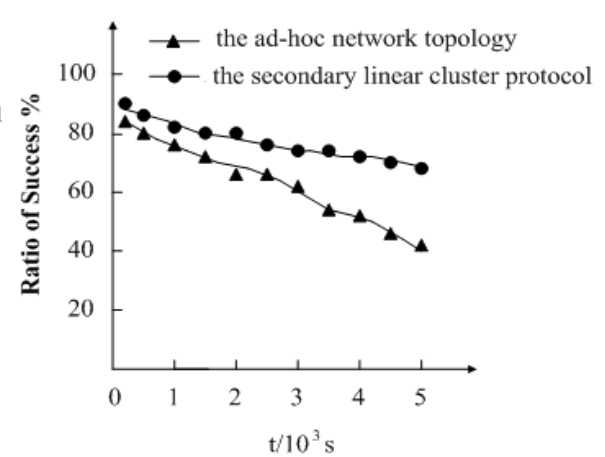

Fig.6 The success rate of data packets

\section{Summary}

According to the characteristic of farmland topography in the Hexi corridor, the distributed two-level linear cluster topology is designed. The cluster nodes of the irrigation sub-network which have not been a cluster head can compete with the principle of equivalent possibility, and on the basis of the rest energy of nodes the cluster head is selected at last. Simulation experiment results show that the distributed two-level linear cluster protocol in the survival of the node and the ratio of success are superior to ad-hoc network. So it can be widely used in the large area which has the same environment characteristics as the Hexi corridor.

No.2015A-163. The supported project - Study of the ZigBee Network Routing Protocol of Water-saving Irrigation of Farmland in the Silk Road Economic Belt.

\section{References}

[1]. Yuanli Liu, Layuan Li, Di Lu: "A Research of energy-efficient cluster-based routing protocol for WSN,” Chinese Journal of Sensors and Actuators, Vol. 23, (2010) No.12, p.1792-1797.

[2]. Yudong Li, Hongguang Huang, Xixi Xiang: "Improved ZigBee routing algorithm based on energy balance,” Computer Engineering and Design, Vol. 32, (2011) No.2, p. 397-400.

[3]. Ruixia Liu, Chunjie Li, Qing Guo: "Cluster routing protocol based on ZigBee mesh network,”Computer Engineering,Vol. 35,(2009) No.3, p. 161-163.

This work was supported by the Foundation Projects in Gansu Province Department of Education 